\title{
Maximum Observed Excretion Rate Normalized by Dose
}

National Cancer Institute

\section{Source}

National Cancer Institute. Maximum Observed Excretion Rate Normalized by Dose. NCI Thesaurus. Code C92396.

The maximum observed excretion rate divided by the dose. 